\title{
REGISTRO PRELIMINAR DEL DEPÓSITO ATMOSFÉRICO ANUAL DE ESPORAS DE HELECHOS DE LAS YUNGAS SOBRE UN GRADIENTE AMBIENTAL EN LA cuenca media del río Perico (Jujuy, Argentina)
}

\author{
GONZALO R. TORRES ${ }^{1}$, CLAUDIO F. PÉREZ², LILIANA C. LUPOํy y OLGA G. MARTINEZ³
}

\begin{abstract}
Summary: Preliminary record of annual atmospheric deposit of fern spores from the Yungas over an environmental gradient in the middle basin of rio Perico (Jujuy, Argentina). The aim of this work was to record the fern spores biological richness, to analyze their relative representation as well as their annual atmospheric deposit rate present in aerobiological traps, on a gradient transition in the Montane Forest of Yungas from Northwest Argentina (1900 m-2600 m a.s.l.). On the altitudinal transect, 5 Tauber traps were placed during a whole year (august 2011-august 2012). A Principal Component Analysis (PCA) was performed to determine the spore assemblages for each altitudinal level. The record includes 11 spore types (Adiantum sp., Anemia sp., Asplenium sp., Cheilanthes squamosa, Microgramma squamulosa, Pecluma sp., Polystichum sp., Pteridaceae indeterminate, Pteris exigua, Pteris deflexa, Pteris inermis) that partially reflect the local flora of the forest understory. Ferns represent $31 \%$ of the total species recorded (both pollen and fern spores), while their relative contribution to the total accumulation rate (4567, 7 propagules $\mathrm{cm}^{-2}$ year-1), was $6.17 \%$ (275.3 spores $\mathrm{cm}^{-2}$ year ${ }^{-1}$ ). The PCA allowed recognizing the spore's associations for each altitudinal level. The study contributes with preliminary deposit rates of fern spores, achieving advances in the knowledge of their aerobiological characteristics. This expands the knowledge of the current environment and serves as reference information for paleoenvironmental studies of the region. The study provides preliminary data on the rate of deposition of fern spores of the Yungas, making progress in the knowledge of the plant group, scarcely known from the aerobiological point of view.
\end{abstract}

Key words: Deposit rate, Spores of ferns, Yungas, Rio Perico.

Resumen: El objetivo del trabajo fue registrar la riqueza de esporas de helechos presentes en trampas aerobiológicas, analizar su representación relativa y la tasa de depósito atmosférico anual, sobre el gradiente de transición entre Selva Montana y Bosque Montano de las Yungas del Noroeste Argentino (1900 m-2600 m s.n.m.). Sobre la transecta altitudinal, se ubicaron 5 trampas Tauber que permanecieron en campo por un año (agosto 2011-agosto 2012). Se realizó un Análisis de Componentes Principales (ACP) para determinar las asociaciones de esporas de helechos de cada nivel altitudinal. El registro incluye 11 tipos de esporas (Adiantum sp., Asplenium sp., Anemia sp., Cheilanthes squamosa, Microgramma squamulosa, Pecluma sp., Polystichum sp., Pteridaceae indeterminada, Pteris exigua, Pteris deflexa, Pteris inermis) que reflejan parcialmente la flora local del sotobosque. Las esporas de helechos representan $31 \%$ del total de especies (polen y esporas), mientras que la abundancia relativa, respecto de la tasa de acumulación total $\left(4567,7\right.$ propágulos $\mathrm{cm}^{-2}$ año-1), representa el $6,17 \%(275,3$ esporas $\mathrm{cm}^{-2}$ año-1). El ACP permitió reconocer las asociaciones de esporas para cada nivel altitudinal. Esto permite ampliar el conocimiento del ambiente actual y sirve como información de referencia para estudios paleoambientales de la región. El estudio aporta datos preliminares sobre la tasa de depósito de esporas de helechos de las Yungas, logrando avances en el conocimiento del grupo vegetal, poco conocido desde el punto de vista aerobiológico.

Palabras clave: Tasa de depósito, Esporas de helechos, Yungas, Río Perico.

\footnotetext{
${ }^{1}$ Centro de Investigación y Transferencia- (CIT-Jujuy), CONICET. Laboratorio de Palinología. Facultad de Ciencias Agrarias, UNJu, Alberdi 47, 4600, San Salvador de Jujuy. Argentina.. Email: gztorres@gmail.com

2 Departamento de Ciencias de la Atmósfera y los Océanos, Facultad de Ciencias Exactas y Naturales, Universidad de Buenos Aires. Pabellón II, $2^{\circ}$ piso, Ciudad Universitaria, (1428), Buenos Aires, Argentina. CONICET

${ }^{3}$ IBIGEO - Herbario MCNS, Facultad de Ciencias Naturales, Universidad Nacional de Salta, Av. Bolivia 5150, 4400, Salta, Argentina.
} 


\section{INTRODUCCIÓN}

Los helechos tienen diversos hábitos (terrestres, epífitos, hemiepífitos, rupícolas o acuáticos) que le permiten crecer en diversos ecosistemas, desde regiones húmedas hasta la periferia de los desiertos (Raunker \& Haufler, 2008). Sin embargo, las comunidades selváticas y boscosas de regiones montañosas concentran la mayor riqueza y diversidad de estas plantas. También, el relieve genera heterogeneidad ambiental, con microclimas y elevaciones que favorecen el establecimiento de diferentes grupos en los distintos pisos altitudinales (Moran, 2008). En el Cono Sur, las Yungas del Noroeste Argentino (NOA) constituyen una de las tres áreas de importancia de concentración de helechos y licófitas (Ponce et al., 2002), donde crecen ca. 200 especies e inclusive se han incorporado nuevos registros en los últimos años (Zuloaga et al., 2008; Larsen et al., 2010; Ganem et al., 2013a).

Si se considera la situación actual de conservación, la flora pteridofítica del NOA está amenazada por actividades humanas como la agricultura, ganadería, deforestación e incendios, entre otras (Giudice et al., 2011). Por otra parte, algunas especies tienen un rol ecológico importante, ya que son pioneras en las primeras etapas de sucesión debido a que tienen la capacidad de colonizar ambientes perturbados, como laderas taladas y bosques recientemente quemados (Dyer \& Lindsay, 1992). En este contexto, el estudio de las esporas constituye una fuente de evidencia valiosa para comprender aspectos de su dispersión, propagación, viabilidad y longevidad (Pérez García \& Reyes Jaramillo, 1993), que a su vez, es indispensable para entender cómo los helechos superan barreras ecológicas y establecen nuevas poblaciones (Kato, 1993). En términos de producción, la cantidad de esporas es variable (entre 100.000 a 30.000 millones por individuo) y su transporte es predominantemente anemófilo, pero también puede ocurrir por acción de otros agentes como el agua o fuerzas físicas como la gravedad y las fuerzas electrostáticas (Kramer et al., 1995, Martínez et al., 2012). Estudios recientes revelaron que la especie nativa de América, Phlebodium aureum (L.) J. Smith, es capaz de liberar las esporas catapultándolas a $10 \mathrm{~m} \cdot \mathrm{s}^{-1}$ (Noblin et al., 2012), mientras que para las esporas de Ophioglossum reticulatum L., Meza Torres et al. (2014) plantearon la posibilidad de transporte intercontinental, entre
África y Sudamérica, a través de las capas bajas de la atmósfera por los sistemas de circulación monzónica. En las regiones tropicales y subtropicales la primera precipitación después de una temporada seca, arrastra las esporas suspendidas en el aire o retenidas en la copa de los árboles ocasionando su depósito (Martínez et al., 2012). Estos son ejemplos de los procesos de despegue y ascenso, transporte horizontal, dispersión lateral y depósito, propuestos por Edmonds (1979) y redefinidos por Isard \& Gage (2001), los que constituyen el modelo fundamental de la Aerobiología y forman el marco conceptual para interpretar el estudio de estos propágulos en el aire.

La metodología aerobiológica permite recopilar información de la diversidad y abundancia de polen y esporas en la atmósfera, reflejando la composición de la vegetación de un área a diferentes escalas espaciales (e.g. La Torre et al., 2010). Asimismo, el monitoreo de bioaerosoles se aplica para detectar cambios en el rango de distribución de las especies nativas, siendo útil como proxy dato de cambios ambientales y de dispersión de especies de interés ecológico, como especies vulnerables, patógenas, invasoras, etc (Benninghoff, 1991, Ferretti, 2009). Estos tipos de estudios también son valiosos para investigaciones paleoambientales del Cuaternario. Las interpretaciones de diversos registros palinológicos fósiles en el NOA y otras regiones de los Andes Centrales, han estimado las condiciones locales y regionales del ambiente en base a la procedencia y distancia de transporte de palinomorfos (Graf, 1992, Lupo, 1998, Schäbitz et al., 2001, Lupo et al., 2006, Torres et al., 2012). Sin embargo, las inferencias paleoambientales realizadas sobre las asociaciones de esporas de helechos, son generales o están limitadas por inconvenientes para establecer su determinación, procedencia y hábitat. Por ello, requieren mayor precisión para definir su valor como indicador de las condiciones ambientales del pasado. La información disponible sobre los helechos está enfocada a descripciones morfológicas de las esporas y/o ecología de la planta (de la Sota, 1977, Morbelli, 1980; Morbelli \& Ponce, 1997, Graf, 1985, Tanco et al., 2009, Morbelli \& Giudice, 2010; Martínez \& Prado, 2011, Martínez et al., 2013, Ganem et al., 2013b) que ayudan a la identificación, pero poco se sabe sobre su presencia y transporte en la atmósfera. Según Rodríguez de la Cruz et al. (2010), 


\section{G. R. Torres et al. - Depósito atmosférico de esporas de Helechos (Jujuy, Argentina)}

las esporas de helechos se encuentran escasamente representadas en el aire en comparación a los granos de polen y esporas fúngicas.

En el área de cerro Negro (Dpto. San Antonio, provincia de Jujuy) se llevaron a cabo estudios preliminares de transporte y depósito polínico arbóreo sobre una transecta altitudinal, utilizando trampas gravimétricas (Torres et al., 2011). En el marco de este estudio, se presentan los primeros datos correspondientes a la composición de esporas de helechos con el objetivo de registrar la riqueza, analizar su representación relativa y la tasa de depósito atmosférico anual, sobre el gradiente ambiental de transición entre la Selva Montana y el Bosque Montano de las Yungas del NOA.

\section{Materiales y Métodos}

\section{Área de estudio}

La cuenca media del río Perico se ubica al Oeste de la localidad de San Antonio, Sur de la provincia de Jujuy (24 $21^{\prime}$ 'S, $65^{\circ} 33^{\prime}$ O, Fig. 1). El valle se extiende en sentido Oeste-Este, abarcando al Oeste la vertiente oriental de la Cordillera Oriental de los Andes, particularmente el sector meridional perteneciente a la sierra del Chañi, y hacia el Este, ocupando estribaciones de las Sierras Subandinas. $\mathrm{El}$ área es drenada por los cauces que vierten, en los colectores principales (río Los Morados - en el Norte - y el del Cerro Negro - al Sur) de cuya confluencia, por debajo de los $2000 \mathrm{~m}$ s.n.m., nace el río de las Cabañas que posteriormente se une al río los Sauces formando el río Perico.
Se trata de una región montañosa que presenta variaciones altitudinales desde los $1300 \mathrm{~m}$ s.n.m., donde se ubica la localidad de San Antonio, hasta las zonas periglaciares de las altas cumbres, que superan los 5000 m s.n.m. (Braun Wilke \& Escalier, 2008).

El clima es subtropical serrano, cálido a templado en el sector medio $(2100$ a $3200 \mathrm{~m}$ s.n.m.) con régimen de precipitación monzónico. Las lluvias se producen por el ascenso de aire húmedo proveniente del Este y se estiman alrededor de los $600 \mathrm{~mm}$ anuales (Bianchi et al., 2005). La temperatura media anual es de $16,1{ }^{\circ} \mathrm{C}$ con variaciones térmicas entre $19^{\circ}-20,9^{\circ} \mathrm{C}$ en verano y entre $10^{\circ}-13,4^{\circ} \mathrm{C}$ en invierno (Braun Wilke et al., 2013). La circulación atmosférica está fuertemente afectada por la topografía predominando los vientos locales de brisas de valle-montaña. Otro tipo de viento local que atraviesa la zona es el llamado "viento norte" que es frecuente en los meses otoñoinvernales por el efecto Föhen (Buitrago, 2000).

La extensión del área, tanto como las variaciones del clima y la topografía, determinan una gran diversidad de flora y vegetación, formando un complejo ambiental de transición entre las provincias fitogeográficas de las Yungas y Altoandina (Cabrera, 1976). El límite de altura inferior, en la cuenca media, lo ocupa la Selva Montana desde los $1500 \mathrm{~m}$ hasta los $2100 \mathrm{~m}$, donde la vegetación dominante se compone de árboles de "Chal-chal" Allophylus edulis (A. St.-Hil., A. Juss. \& Cambess). Hieron. ex Niederl., "Laurel de la falda" Cinnamomum porphyrium Griseb. Kosterm, "Horco molle" Blepharocalix salicifolius Kunth O. Berg, "Cochucho" Zanthoxylum coco Gillies ex Hook. f. \& Arn., entre otros. A partir

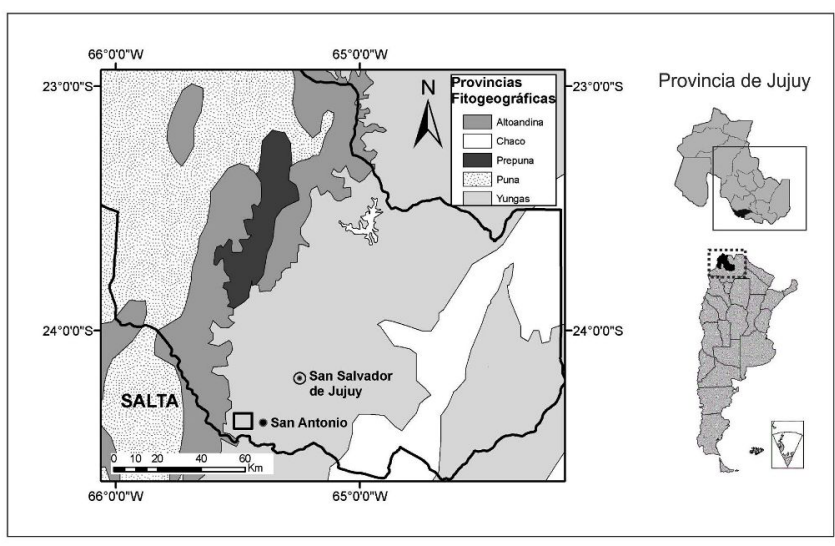

Fig. 1. Mapa de ubicación del área de estudio. Departamento San Antonio, Provincia de Jujuy, Argentina. 
de los 2100 m comienza el Bosque Montano, donde las principales especies leñosas que lo integran son el "Aliso del cerro" Alnus acuminata Kunth, "Nogal criollo" Juglans australis Griseb., "Tala" Celtis ehrembergiana Klotzsch Liebm. y "Molulo" Sambucus nigra L. ssp peruviana Kunth R. Bolli. El Bosque Montano alcanza su límite superior a los $2600 \mathrm{~m}$, formando un bosque homogéneo de Aliso. A continuación se extiende el Pastizal Altoserrano hasta los $3500 \mathrm{~m}$, constituido por comunidades de pastos duros de crecimiento fasciculado que incluye Poáceas como Festuca hieronymi Hack., Deyeuxia spp., Paspalum spp.y Stipa spp, entre otras. Por encima del pastizal, se establece una transición hacia la estepa herbácea altoandina (Braun Wilke \& Escalier, 2008). La Asociación Bosque Modelo Jujuy (BMJ) realizó estudios complementarios de vegetación a través del relevamiento de la flora pteridofítica, donde han registrado hasta el momento ca. 50 especies (BMJ, inédito).

\section{Muestreo del depósito atmosférico}

Los datos de concentración atmosférica se tomaron utilizando la metodología propuesta por Tauber (1974). La preparación de los captadores para la exposición en campo consistió en el agregado de cristales de fenol, para evitar el crecimiento de hongos y algas. El volumen del recipiente del captador se calculó considerando la precipitación media anual de la estación meteorológica más cercana para evitar pérdida de material por desborde.

Sobre una transecta altitudinal, comprendida entre los $1900 \mathrm{~m}$ y $2600 \mathrm{~m}$ s.n.m., se ubicaron 5 estaciones permanentes de muestreo separadas aproximadamente cada $210 \mathrm{~m}$ de altura. Las mismas se colocaron a 1,2 $\mathrm{m}$ sobre el suelo, en lugares relativamente despejados para garantizar flujos de aire poco perturbados y su posición y altitud se determinó con GPS. Los captadores permanecieron en el campo por el término de un año (agosto 2011-agosto2012) con la finalidad de obtener la tasa anual de depósito de los propágulos. Al concluir el tiempo de exposición, los captadores fueron retirados para el procesamiento de las muestras en laboratorio y conteo del material colectado. El procesamiento de las muestras se realizó en el Laboratorio de Palinología de la Facultad de Ciencias Agrarias (UNJu) mediante técnicas estándar de extracción, consistente en filtrado con malla de 250 $\mu \mathrm{m}$, incorporación de una pastilla de Lycopodium clavatum (Stokmarr, 1971) para el cálculo de concentración absoluta, eliminación de materia orgánica con $\mathrm{KOH}$ al 10\%, acetólisis (Erdtman, 1960), lavados con ácido acético y montaje con parafina. El conteo se realizó con microscopio óptico Leica DM500 con un aumento de 400x. Se contaron un mínimo de 250 propágulos por preparado, incluyendo polen y esporas. La determinación se llevó a cabo a través de atlas palinológicos (Heusser, 1971, Morbelli, 1980, Tryon \& Lugardon, 1991) y por comparación con la esporoteca del Museo de Ciencias Naturales de Salta. Las fotografías fueron tomadas con cámara Leica ICC50 incorporada al microscopio. Los datos de tasa de depósito atmosférico se expresan en $\mathrm{N}^{\circ}$ de esporas $\mathrm{cm}^{-2}$ año $^{-1}$ y se presenta un diagrama elaborado con el programa PanPlot versión 11.04 (Sieger et al., 2005). Con la matriz de datos de tasa de depósito se elaboró un análisis de componentes principales (ACP) para determinar las asociaciones de esporas de helechos de cada nivel altitudinal. El procesamiento se llevó a cabo con el software estadístico Infostat versión 2011 (Di Rienzo et al., 2011).

\section{Resultados}

La composición general de la nube esporopolínica del área de estudio, muestra una riqueza integrada por 12 tipos polínicos arbóreos (AP), 13 tipos polínicos no arbóreos (NAP) y 11 tipos de esporas de helechos, entre las cuales se incluyen especies terrestres y epífitas (Tabla 1, Fig. 2).

De la riqueza total de propágulos colectados, el contenido de esporas de helechos expresa una representación relativa del 31\% (Fig. 3a), mientras que su abundancia relativa, respecto de la tasa de acumulación total $\left(4567,7\right.$ propágulos $\mathrm{cm}^{-2}$ año $\left.{ }^{-1}\right)$, solo representa el 6,17 \% (275,3 esporas $\mathrm{cm}^{-2}$ año $^{-1}$, Fig. 3b).

Se observa que tanto las asociaciones como la tasa de depósito de los diferentes taxones varían en cada nivel altitudinal (Fig. 4). El género Polystichum fue el mejor representado, mostrando la distribución más amplia y las mayores tasas de depósito, alcanzando el mínimo de 11,7 y un máximo de 49,5 esporas $\mathrm{cm}^{-2}$ año $^{-1}$, a los $2100 \mathrm{y}$ $2600 \mathrm{~m}$ respectivamente. El segundo género de mayor distribución es Anemia, cuyos valores varían entre un máximo de 12,28 esporas $\mathrm{cm}^{-2}$ año ${ }^{-1}$ en 
la Selva Montana y decrece a 10,97 esporas $\mathrm{cm}^{-2}$ año ${ }^{-1}$ en el bosque homogéneo de Aliso del cerro. La presencia de las restantes esporas se encuentra restringida a uno o dos niveles altitudinales y muestran una tasa de depósito variable. Las tasas de depósito más bajas pertenecen a Cheilanthes squamosa y Pteridaceae indeterminada, las cuales no superan las 7 esporas $\mathrm{cm}^{-2}$ año ${ }^{-1}$.

El ACP (Fig. 5) señala dos componentes principales que explican $72,2 \%$ de la variabilidad de los datos. El CP 1 (38,8\%) diferencia las asociaciones de esporas de los pisos altitudinales inferiores (1900 a $2450 \mathrm{~m}$ s.n.m.) que presentan la mayor influencia en la distribución de los taxones. A su vez, este componente discrimina dos conjuntos de asociaciones de esporas, correspondientes a la Selva Montana (A) y al Bosque Montano (B). El CP $2(33,4 \%)$ separa un tercer conjunto

Tabla 1. Composición de la nube esporopolínca del área de estudio. (1) Helecho terrestre. (2) Helecho epífito.

\begin{tabular}{|c|c|c|c|}
\hline Taxón & Polen Arbóreo (AP) & Polen no arbóreo (NAP) & Helecho \\
\hline Adiantum sp. (1) & & & $\mathrm{X}$ \\
\hline Alnus acuminata Kunth & $\mathrm{X}$ & & \\
\hline $\begin{array}{l}\text { Allophylus edulis (A. St.-Hil., A. Juss. \& Cambess). } \\
\text { Hieron. ex Niederl }\end{array}$ & $\mathrm{X}$ & & \\
\hline Amaranthaceae- Chenopodiaceae & & $\mathrm{x}$ & \\
\hline Anacardiaceae & $\mathrm{X}$ & & \\
\hline $\begin{array}{l}\text { Anadenanthera colubrina (Vell) Brenan cebil (Griseb) } \\
\text { Altschul }\end{array}$ & $\mathrm{x}$ & & \\
\hline Anemia sp. (1) & & & $\mathrm{X}$ \\
\hline Asplenium sp. (1) & & & $x$ \\
\hline Asteraceae & & $\mathrm{X}$ & \\
\hline Caryophyllacae & & $\mathrm{x}$ & \\
\hline Celtis sp. & $\mathrm{x}$ & & \\
\hline Cheilanthes squamosa Gilles ex Hook \& Grev (1) & & & $\mathrm{X}$ \\
\hline Cyperaceae & & $\mathrm{X}$ & \\
\hline Euphorbiaceae (Tipo Sapium) & $\mathrm{X}$ & & \\
\hline Fabaceae & & $\mathrm{X}$ & \\
\hline Hyptis sp. & & $\mathrm{x}$ & \\
\hline Juglans australis Griseb. & $x$ & & \\
\hline Lauraceae (Tipo Phoebe) & $\mathrm{X}$ & & \\
\hline Malvaceae & & $\mathrm{X}$ & \\
\hline Mimosa sp. & & $\mathrm{x}$ & \\
\hline Microgramma squamulosa (Kaulf.) de la Sota (2) & & & $\mathrm{X}$ \\
\hline Myrtaceae (Tipo Blepharocalix) & $x$ & & \\
\hline Parapiptadenia excelsa (Griseb.) Burkart & $\mathrm{X}$ & & \\
\hline Pecluma sp.(2) & & & $\mathrm{x}$ \\
\hline Pinus sp. & $x$ & & \\
\hline Podocarpus parlatorei Pilg & $x$ & & \\
\hline Poaceae & & $\mathrm{X}$ & \\
\hline Polystichum sp.(1) & & & $\mathrm{x}$ \\
\hline Pteridaceae indeterminada(1) & & & $\mathrm{X}$ \\
\hline Pteris exigua O.G. Martínez \& J Prado (1) & & & $\mathrm{X}$ \\
\hline Pteris deflexa Link (1) & & & $\mathrm{X}$ \\
\hline Pteris inermis (Rosenst) de la Sota (1) & & & $\mathrm{X}$ \\
\hline Rosaceae & & $x$ & \\
\hline Urticaceae & & $\mathrm{X}$ & \\
\hline Verbenaceae & & $\mathrm{X}$ & \\
\hline Zanthoxylum coco Gilles ex Hook f \& Arn & $\mathrm{X}$ & & \\
\hline
\end{tabular}


Bol. Soc. Argent. Bot. 49 (4) 2014

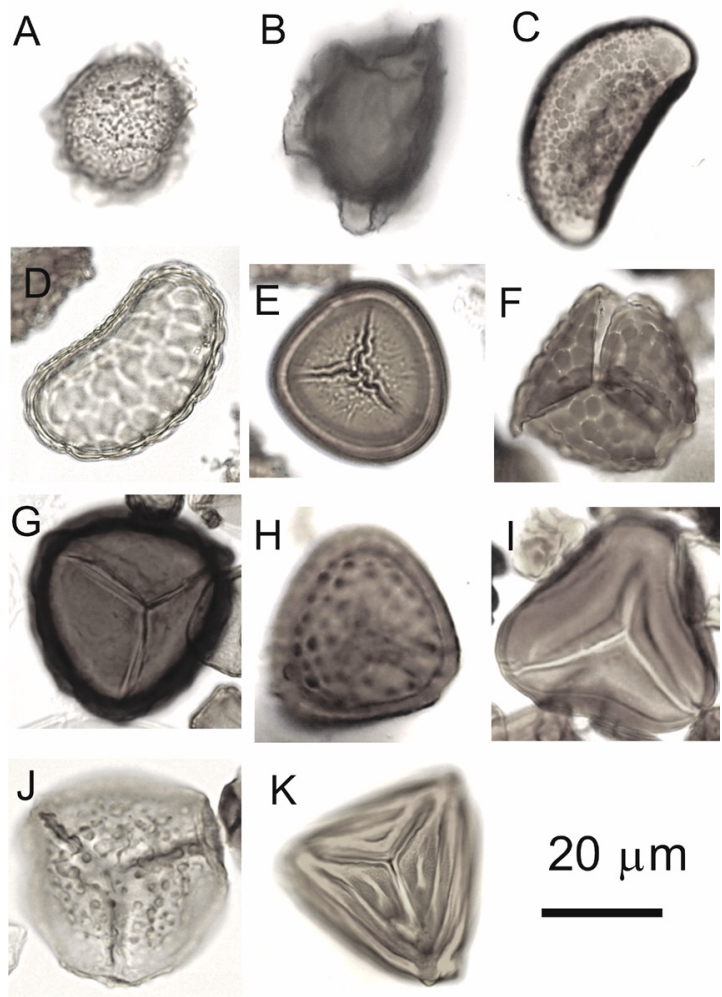

Fig. 2. Riqueza de esporas presentes en trampas Tauber. A: Aspleniaceae: Asplenium sp. B: Dryopteridaceae: Polystichum sp. C-D: Polypodiaceae. C: Microgramma squamulosa. D: Pecluma sp. E-J: Pteridaceae. E: Adiantum sp. F: Cheilanthes squamosa. G: Pteris exigua. H: Pteris deflexa. I: Pteris inermis. J: Pteridaceae indeterminada. K: Schizaeaceae: Anemia sp.

(C) perteneciente a el piso superior $(2600 \mathrm{~m})$, caracterizando una asociación diferente de esporas.

\section{Discusión y Conclusiones}

Los resultados señalan que la riqueza de esporas registradas refleja la composición parcial de la flora pteridofítica local (Ponce et al., 2002, Zuloaga et al., 2008), perteneciente a las comunidades del sotobosque.

A pesar de la gran cantidad de esporas que pueden producir los helechos (Kramer et al., 1995, Martínez et al., 2012), el registro muestra que la abundancia relativa (Fig. 3b) es menor en comparación con los granos de polen con síndrome

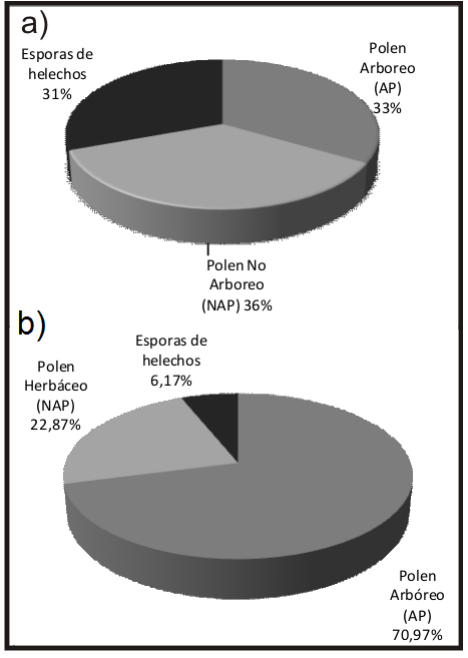

Fig. 3. Evaluación de la presencia de las esporas de helechos en la atmósfera del área de estudio. a) Representación relativa. b) Abundancia relativa.

de polinización equivalente. Esta situación podría deberse a las propiedades intrínsecas de las esporas de los helechos, como forma, tamaño y peso ya que estas condicionan sus propiedades aerodinámicas y el proceso de transporte, haciendo que el mismo tenga en general menor alcance en comparación a los granos de polen.

Un aspecto significativo del registro, es que se han encontrado en el aire del área de estudio, esporas pertenecientes a plantas no registradas en los relevamientos de vegetación (BMJ, inédito), como Pteris deflexa, P. exigua, P. inermis y Pecluma sp. Según Tanco et al., 2009, Martínez \& Prado, 2011, Martínez et al., 2013 y Giudice et al., 2004, las especies mencionadas tienen una distribución y ecología que indican que el ambiente del área es favorable para su crecimiento. Por lo tanto, no se descarta que estén presentes en la vegetación pero que aún no hayan sido detectadas. Estas especies son importantes por ser endémicas (Martínez \& Prado, 2011) y por su vulnerabilidad desde el punto de vista de la conservación (Giudice et al., 2011). En este sentido, se considera que la metodología empleada resultó una herramienta eficiente para revelar la presencia de especies de importancia ecológica (Benninghoff, 1991, Ferretti, 2009).

El helecho Cheilanthes squamosa, ha sido citado para la provincia en ambientes de pastizales serranos, 


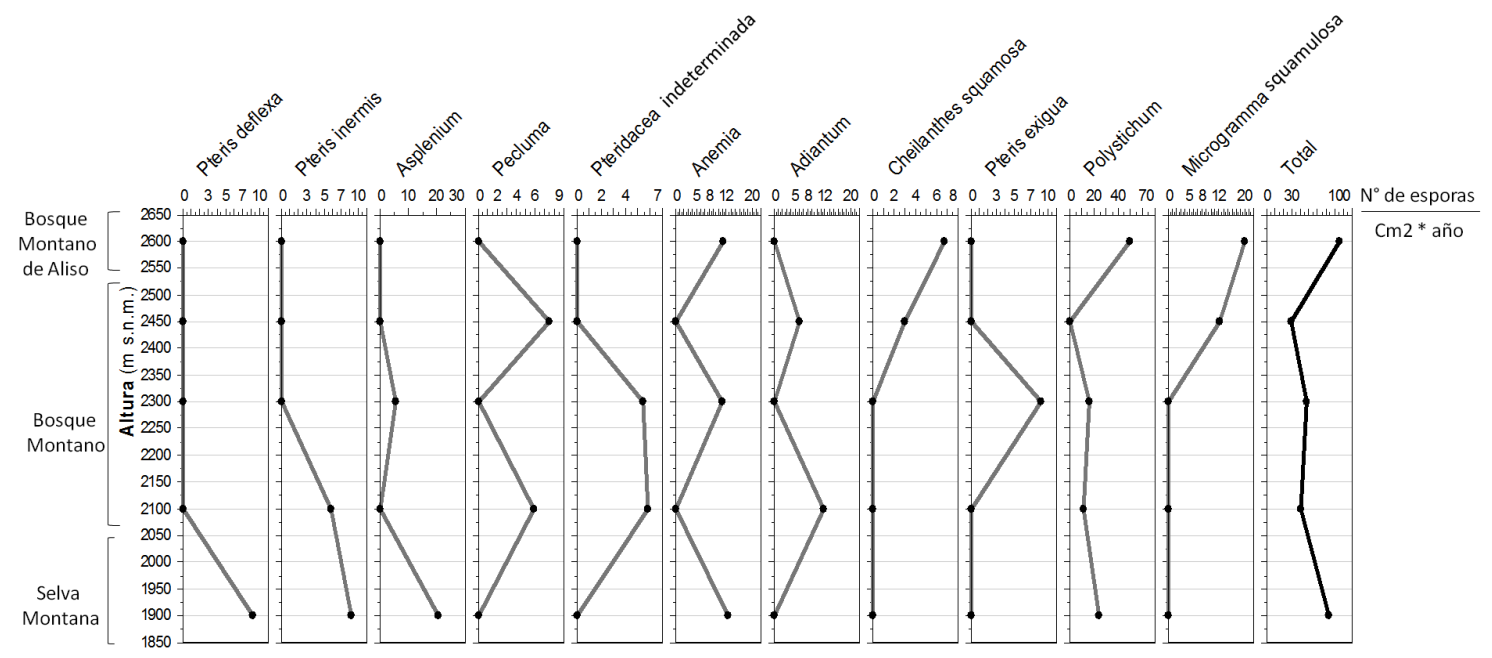

Fig. 4. Tasa de depósito atmosférico anual de esporas de helechos.

pudiendo crecer entre los 2000 y 4500 m s.n.m. (de la Sota, 1977). La espora de esta especie presenta una tasa de depósito baja y los valores disminuyen con la altura (Fig. 4). Por ello, se considera que el aporte posiblemente provenga del piso de vegetación adyacente al bosque de Aliso del cerro, el Pastizal Altoserrano. Consecuentemente, se plantea la posibilidad que su presencia en pisos altitudinales inferiores podría responder al transporte inducido por circulaciones atmosféricas locales (Buitrago, 2000), particularmente durante las condiciones de viento catabático, el cual desciende por la ladera del valle a partir de horarios vespertinos.

Sobre la base del ACP(Fig. 5) se pueden establecer asociaciones altitudinales de contenido atmosférico de esporas, permitiendo caracterizar cada ambiente. La Selva Montana (1900 m) se caracteriza por la presencia de Asplenium sp., Pteris deflexa, Pteris inermis y Anemia sp. El sector del Bosque Montano $(2100$ y $2450 \mathrm{~m})$ se define por la asociación de Pecluma sp., Adiantum sp. y Pteridaceae. Finalmente para el Bosque de Aliso del cerro (2600 m), se vinculan Microgramma squamulosa, Polystichum sp. y Cheilanthes squamosa. Es decir que el gradiente ambiental estudiado en la zona, puede caracterizarse por el ensamble de esporas de helechos presentes en la atmósfera. Estos resultados ayudan a mejorar las interpretaciones paleoambientales sobre las asociaciones de esporas que puedan estar presentes en registros fósiles de sitios ubicados en la región Cordillerana del NOA, ya que brinda un marco de referencia que ayuda a clarificar los interrogantes vinculados a la procedencia de esporas del sotobosque de los diferentes pisos de vegetación de las Yungas. Así, la información adquirida enriquece el conocimiento del ambiente actual, aportando datos a estudios que tratan los problemas relacionados a los cambios ecológicos del pasado a escala regional. También, se abre la oportunidad de continuar las investigaciones para complementar los resultados obtenidos, ya que aún se deben caracterizar las condiciones meteorológicas asociadas al transporte e indagar en el valor indicador de las esporas que aún no han sido abordado en detalle en los estudios paleoambientales de la región (Lupo, 1998, Schäbitz et al., 2001, Lupo et al., 2006, Torres et al., 2012).

Conocer las tasas de depósito y el alcance del transporte de las esporas, es de vital importancia para comprender la distribución geográfica (áreas disyuntas, conexiones en el pasado, rutas migratorias) y mecanismos de especiación, entre otros aspectos.

\section{Agradecimientos}

A los proyectos Secter-Unju A0-110 y PICT 2010-0554 por la financiación parcial. A la asociación Bosque Modelo Jujuy por el apoyo logístico en las campañas, a los pobladores de 
Bol. Soc. Argent. Bot. 49 (4) 2014

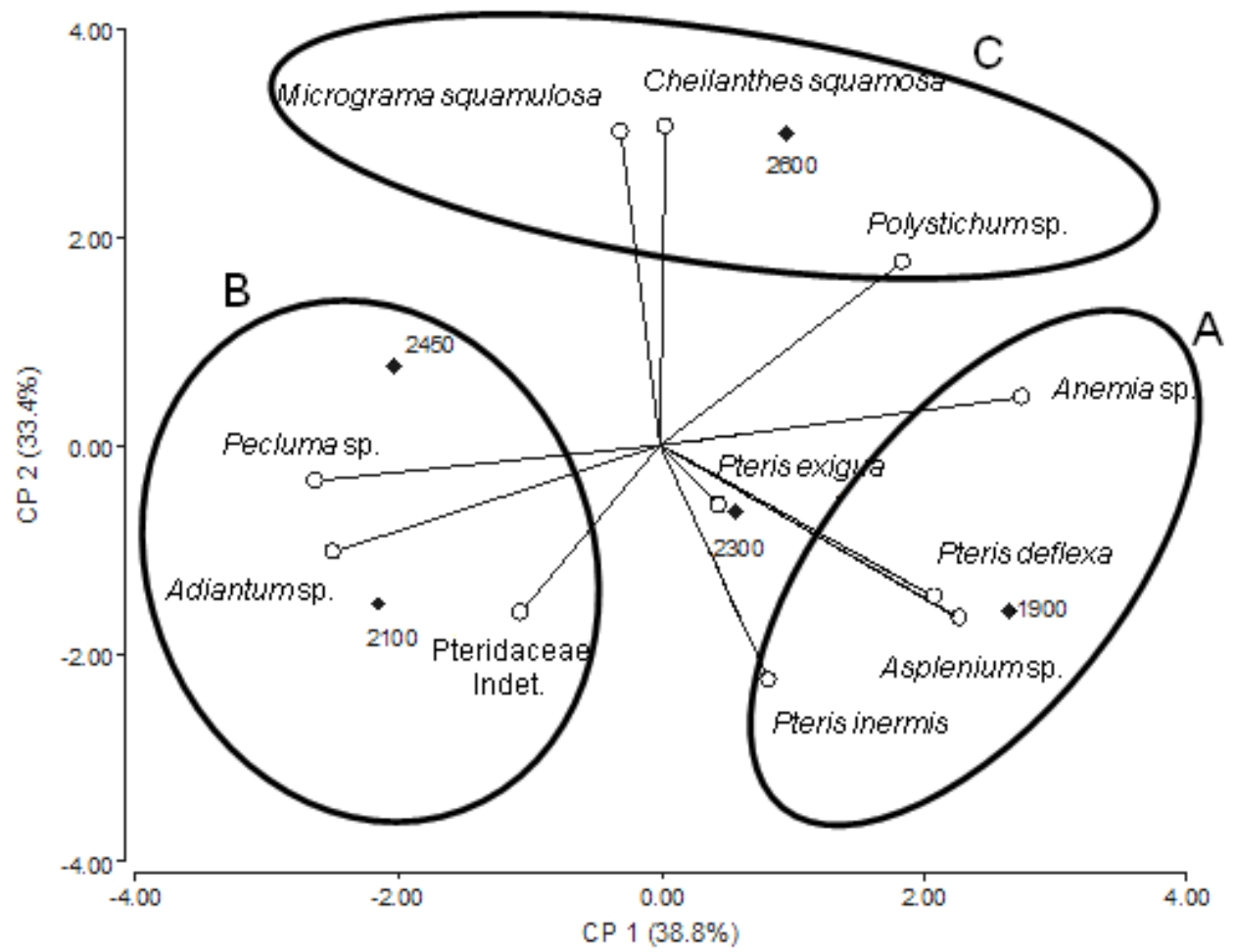

Fig. 5. Análisis de componentes principales. Los conjuntos A, B y C señalan las asociaciones de esporas características de los pisos altitudinales. A: asociación de Selva Montana. B: Asociaciones de Bosque Montano. C: Asociación del Bosque Montano de Aliso.

Cerro Negro por hospedarnos durante los trabajos de campo y al Ing. Agr. Lauro Cazón por la ayuda con el programa estadístico. A los revisores por las contribuciones al manuscrito.

\section{Bibliografía}

BENNINGHOFF, W. 1991. Aerobiology and its significance to Biogeography and Ecology. Grana 30: $9-15$.

BIANCHI, A., C. YAÑEZ \& L. ACUÑA. 2005. Base de datos mensuales de precipitaciones del Noroeste Argentino. Ed. INTA. EEA. Salta-Jujuy. 41 pp

BRAUN WILKE. R., E. SANTOS, L. PICCHETTI, M. LARRÁN, G. GUZMÁN, C. COLARICH \& C. CASOLI. 2013. Carta de aptitud ambiental de la provincia de Jujuy. Ediunju. San Salvador de Jujuy. $269 \mathrm{pp}$.

BRAUN WILKE, R. \& C. I. ESCALIER. 2008. Estudio de vegetación en la Finca Cerro Negro. Informe Proyecto "Precodep"- Bosque Modelo Jujuy.
BUITRAGO, L.G. 2000. El Clima de la provincia de Jujuy. 2da Ed. Cátedra de Climatología y Fenología. Ed UNJu. Jujuy. 39 pp.

CABRERA, A. 1976. Provincias fitogeográficas de Argentina. Enciclopedia Argentina de Agricultura y Jardinería. Ed. Acme. Buenos Aires.

DE LA SOTA, E. R. 1977. Pteridófitas, en A. L. Cabrera (ed.), Flora de la Provincia de Jujuy, República Argentina. Colección Científica del Instituto Nacional de Tecnología Agropecuaria. 13: 1- 275.

DI RIENZO, J.A., F. CASANOVES, M.G. BALZARINI, L. GONZÁLES, M. TABLADA \& C.W. ROBLEDO. 2011. InfoStat. Universidad Nacional de Córdoba.

DYER, A.F. \& S. LINDSAY. 1992. Soil spore banks of temperate ferns. Amer. Fern J. 82: 89-122.

ERDTMAN, G. 1960. The acetolysis method. Svensk. Bot. Tidskr. 54: $561-564$.

FERRETTI, M. 2009. Quality assurance in ecological monitoring - towards a unifying perspective. $J$. Environ. Monit.11: 726-729.

GANEM, M. A., J. P. RAMOS GIACOSA, M. L.LUNA, M. ARANA, A. ROTMAN, O. AHUMADA, E.R. DE LA SOTA \& G.E. GIUDICE. 2013a. Diversidad 


\section{G. R. Torres et al. - Depósito atmosférico de esporas de Helechos (Jujuy, Argentina)}

de helechos y licofitas del Parque Nacional Calilegua, Provincia de Jujuy, Argentina. Bol. Soc. Argent. Bot. 48: 567-584.

GANEM, M. A, M. L. LUNA \& G. E. GIUDICE. 2013 b. Estudio palinológico en especies de Asplenium (Aspleniaceae) de Argentina. Bol. Soc. Argent. Bot. 48: 465-476.

GIUDICE, G. E, M. A. MORBELLI, M. R. PIÑEIRO, M. COPELLO \& G. ERRA. 2004. Spore morphology of the Polypodiaceae from Northwestern Argentina. Amer. Fern J. 94: 9-27.

GIUDICE, G. E., J. P. RAMOS GIACOSA, M. L. LUNA, C. MACLUF, M. PONCE, G. MÁRQUEZ \& E. R. DE LA SOTA. 2011. Evaluación preliminar del grado de amenaza de los helechos y licofitas de Argentina. Bol. Soc. Argent. Bot. 46: 151-161.

GRAF, K. 1985.Esporas triletes de helechos bolivianos. Boletín del Servicio Geológico de Bolivia. Serie A. Vol III No.1:33-55

GRAF, K. 1992. Pollensiagramme aus den Anden. Eine Synthese zur Klimageschichte und Vegetationsentwicklung seit der letzten Eiszeit. Physusche Geographie. Vol 34. 138 pp.

HEUSSER, C. J. 1971. Pollen and spores of Chile. The University of Arizona Press. Tucson. 167 pp.

KATO, M. 1993. Biogeography of ferns: Dispersal and vicariance. Journal of biogeography 20: 265-274.

KRAMER K. U., J. J. SCHNELLER \& E. WOLLENWEBER. 1995. Farne und Farnverwandte, Erste Auflage. Thieme Verlag, Stuttgart, Germany. 198 pp.

LARSEN, C., O. G. MARTÍNEZ \& M. PONCE. 2010. Nuevos registros en helechos para el noroeste de Argentina. Darwiniana 48: 100-108.

LATORRE, F, C. F. PÉREZ, S. STUTZ \& S. PASTORINO. 2010. Pollen deposition in tauber traps and surface soil samples in the Mar Chiquita costal lagoon area, pampa grassland (Argentina). Bol. Soc. Argent. Bot. 45: 321-332.

LUPO, L. C. 1998. Estudio sobre la lluvia polínica actual y la evolución del paisaje a través de la vegetación durante el Holoceno en la cuenca del río Yavi. Borde oriental de la puna, Noroeste argentino. Tesis Doctoral. Bamberg. Alemania.

LUPO, L. C; M. M. BIANCHI, E. ARÁOZ, R. GRAU, CH. LUCAS, R. KERN, M. CAMACHO, W. TANNER \& M. GROSJEAN. 2006. Climate and human impact during the past 2000 years as recorded in the Lagunas de Yala, Jujuy, northwestern Argentina. Quaternary International. 158: 30-43.

MARTÍNEZ, O. G. \& J. PRADO. 2011. Pteris exigua (Pteridaceae), a new endemic specie from Tucumano-Boliviano forest in northwet Argentina. Brittonia 63: 295-299.
MARTÍNEZ, O. G., M. E. TANCO, J. CHAMBI, M. C. BONOMO, M. S. RAMÍREZ \& Z. AVILEZ. 2012. Sobre la biología reproductiva de los helechos. Temas de Biología y Geología del NOA. 2: 48-53.

MARTÍNEZ, O. G., C. PRADA, M. E. TANCO \& M. C. BONOMO. 2013. Sexual phase of three species of Pteris (Pteridaceae). Tropical Plant Biol. 6:46-52

MEZA TORRES, E. I, B. CERNE, A. G. ULKE \& M. A. MORBELLI. 2014. Distribution of Ophioglossum reticulatum L. in South America.

A case of long-distance jump dispersal? Int J Biometeorol. DOI 10.1007/s00484-014-0830-8.

MORAN, R. C. 2008. Diversity, Biogeography, and Floristic. In. T.A. Ranker and C.H. Haufler eds. Biology and Evolution of Fern and Lycophytes. Cambridge University Press, 367-394.

MORBELLI, M. A. 1980. Morfología de las esporas de Pteridophyta presentes en la región fuegopatagónica. República Argentina. Opera lilloana 28: $1-138$.

MORBELli, M. A. \& M. M. PONCE. 1997. Palynological study of Cheilanthes and Astrolepis (Pteridaceae) species from Northwestern Argentina. Amer. Fern J. 87: $51-65$.

MORBELli, M. A. \& G. E. GIUDICE. 2010. Spore wall ultrastructure of Polypodiaceae from northwestern Argentina. Grana 49: 204 - 214.

NOBLIN, X., N. O. ROJAS, J. WESTBROOK, C. LLORENS, M. ARGENTINA, J. DUMAIS. 2012. The fern sporangium: a unique catapult. Science 335: 1322-1322.

PÉREZ GARCÍA, B. \& I. REYES JARAMILLO. 1993. Helechos: propagación y conservación. Ciencias 30:11-17.

PONCE, M., K. MEHLTRETER \& E. R. DE LA SOTA. 2002. Análisis biogeográfico de la diversidad pteridofítica en Argentina y Chile continental. Revista Chilena de Historia Natural 75: 703-717.

PONCE, M. \& O. G. MARTÍNEZ. 2012. Dryopteridaceae. Flora del Valle de Lerma.

RANKER, T. \& CH. HAUFLER (eds.). 2008. Biology and Evolution of Ferns and Lycophytes. Cambridge University Press, Cambridge.

RODRIGUEZ DE LA CRUZ, D., E. SÁNCHEZ-REYES \& J. SÁNCHEZ-SÁNCHEZ. 2010. Aerobiology of pteridohyta spores: Preliminary results and application. En KUMAR, A., H. FERNANDEZ \& M. A. REVILLA (eds). Working with ferns. Springer. New York. 217-281 pp.

SCHÄBITZ, F., L. LUPO, J. A. KULEMEYER, \& J. J. KULEMEYER. 2001. Variaciones en la vegetación, el clima y la presencia humana en los ultimos 15.000 años en el Borde Oriental de la Puna. Provincias de Jujuy y Salta, noroeste argentino. APA. Publicación especial 8: 155-162. 
SIEGER, R.; H. GROBE \& M. DIEPRENBOEK. 2005. PanPlot - software to visualize profiles and core logs. Alfred Wegener Institute, Helmholtz Center for Polar and Marine Research, Bremerhaven, doi:10.1594/PANGAEA.330147.

STOCKMARR, J. 1971. Tablets with spores used in absolute pollen analysis. Pollen et Spores. 13: 615-621.

TANCO, M. E., O. G. MARTÍNEZ \& M. L. C. BONOMO. 2009. Germinación y morfogénesis gametofítica de Pteris inermis (Rosent.) de la Sota. Gayana Bot. 66: 10-17.

TAUBER, H. 1974. A static non-overload pollen collector. The New Phytologist. 73:359-369.

TORRES, G. R., L. C. LUPO \& C. F. PÉREZ. 2011. Transporte y depósito polínico arbóreo entre las Sierras Subandinas y la Cordillera Oriental de Jujuy. Bol. Soc. Argent. Bot. 46: 265.

TORRES, G. R., L. C. LUPO \& C. F. PÉREZ. 2012. Estudio paleoecológico de los humedales andinos de
Cerro Negro (Jujuy, Argentina) durante el Holoceno Tardío: Resultados preliminares. Resumen. XV Simposio de Paleobotánica y Palinología. Corrientes. Argentina.

TRYON, A. F. \& B. LUGARDON. 1991. Spores of the Pteridophyta. New York: Springer-Verlag.

ZULOAGA, F. O., O. MORRONE \& M. J. BELGRANO (eds.) 2008. Catálogo de las Plantas Vasculares del Cono Sur. Vol. 1. Pteridophyta, Gymnospermae, Monocotyledoneae. Monographs in Systematic Botany from the Missouri Botanical Garden 107. Saint Louis: Missouri Botanical Garden.

Recibido el 27 de diciembre de 2013, aceptado el 28 de agosto de 2014. 